\title{
AN EFFECTUAL EMOTION RECOGNITION SYSTEM BASED ON FUZZY LOGIC
}

\author{
Er. Vibha Dutta ${ }^{1}$, Dr. R. C. Gangwar ${ }^{2}$ and Er. Mohit Marwaha ${ }^{3}$
}

\begin{abstract}
Despite the fact that sharing pictures and recordings are well known, messages and blog articles still remain as an essential part to express feelings. Contrasted with exploration of feeling identification in multimodal fields, sentiment discovery from text is still unexplored and requires more changes to be amassed as useful applications. That is why need of effectual classification algorithm is very much required these days. This paper presents the work that depends on execution of fuzzy logic in perceiving emotions from text. The work is based on implementation of fuzzy logic in recognizing emotions from text in MATLAB environment.

Keywords - Emotion recognition, MATLAB, Fuzzy logic.
\end{abstract}

\section{INTRODUCTION}

Use of social media is increasing day-by-day. Social media growth leads to the usage of internet as the participation of persons is increasing simultaneously. In case of product review, user helps a lot about decision making of the product $[11,12,13]$. But manually reading such a bulk amount review is a very difficult task. So, there is a need of an automatic system, which will lead to automatically extract the positive and negative features of the product and make the decision making process easier. There are many sites and companies, which perform these activities [16]. In this work, sentiment analysis of big data will be done using fuzzy logic and SVM. Detecting emotional state of a person by analyzing a text document written by him/her appears challenging but is essential many times due to the fact that most of the times textual expressions are not only using emotion words but also result from the interpretation of the meaning of concepts and interaction of concepts which are described in the text document. Recognizing the emotion of the text plays a key role in the human-computer interaction [17]. Emotions may be expressed by a person's speech, face expression and written text known as speech, facial and text based emotion respectively. Sufficient amount of work has been done related to speech and facial emotion recognition but text based emotion recognition system still needs attention of researchers. In computational linguistics, the detection of human emotions in text is becoming increasingly important from an applicative point of view.

Emotion is expressed as joy, sadness, anger, surprise, hate, fear and so on. Since, there is not any standard emotion word hierarchy; main focus is on the related research about emotion in cognitive psychology domain. In 2001, W. GerrodParrot, composed a book named "Emotions in Social Psychology", wherein he clarified the Sentiment framework and formally arranged the human Sentiments through a Sentiment chain of command in six classes at essential level which are Love, Joy, Anger, Sadness, Fear and Surprise. Certain different words additionally fall in optional and tertiary levels. Now, scientists have concentrated on this region [19] and

\footnotetext{
${ }^{1}$ Department of Computer Science and Engineering Bcet, Punjab,India

${ }^{2}$ Department of Computer Science and Engineering Bcet, Punjab,India

${ }^{3}$ Department of Computer Science and Engineering Bcet, Punjab,India
} 
attempting to get sentiment data to examine and abridge the operation inions communicated naturally with PCs. This new research area is generally called Opinion mining of enormous information and Sentiment Analysis [18].

Classification problem is one of the problem in which data has to be classified into different classes present inside the $\mathrm{db}$. This extricated data can be then further arranged by extremity as positive, negative or impartial. It can be characterized as a computational errand of separating notions from the supposition. A few suppositions speak to conclusions and a few assessments don't speak to any opinion [19].

Sentiment analysis (SA) is also known as sentiment mining of huge information. SA is the computational investigation of Opinions, conclusions, subjectivity toward an element. The element represents the people, occasions or points. The essential work in the information mining can be arranged in two consequent ways. First is called grouping and the other is called making clusters. Information Mining is a term of seeking and investigates of information. Fuzzy logic is thoughtfully straightforward.

There are countless media sites that empower clients to contribute, adjust and grade the content. Clients have a chance to express their own assessments about particular themes. The case of such sites incorporate web journals, gatherings, item audits destinations, and interpersonal organizations. The proposed methodology in this work will utilize the common dialect handling techniques like fuzzy logic to extract emotions from text.

Fuzzy logic is easy to apply and understand. Mathematical concepts of fuzzy logic is also very simple. It is based on the natural language.

\section{USE OF SOCIAL MEDIA}

About four out of five clients of web use online networking for few or other connection. Some of these incorporate kinship systems, blogging and video sharing destinations, ebusiness locales and so on [20]. The inclusion and commitment of the clients on the web is expanding step by step. One such commitment is audits of clients in long range interpersonal communication destinations. The present pattern of giving online surveys empowers clients to take better choices who need to utilize a specific administration or buy a specific item. It helps them to check the prominence of the item. It likewise empowers them to extricate the positive or negative elements of the items by perusing surveys [21].Manual examination of such an enormous measure of audits can prompt one-sided choice. So, to give computerization, we are examining assumption examination. Supposition examination is the advanced technique which dissect enormous measure of information to concentrate assessments connected with the information. The development of web has an extraordinary criticalness in online administration.

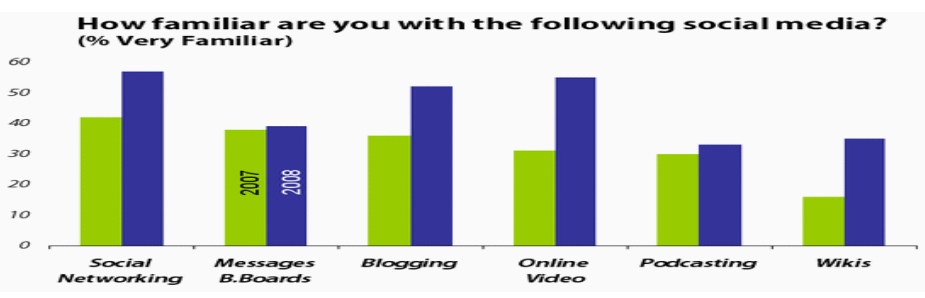

Figure. Error! No text of specified style in document. Depicting the Users of each type of social media platform 


\section{A. Type of Social Media Applications}

There are many social media platforms which has become an integral part of people's lives these days. Some of the famous and most popular among them are:

\begin{tabular}{ll} 
- & Facebook \\
- & Twitter \\
- & Linkedin \\
- & Guora \\
- & GooglePlus \\
\hline & Youtube14
\end{tabular}

The patter on of users in the specific platforms is shown in Figure. 2.

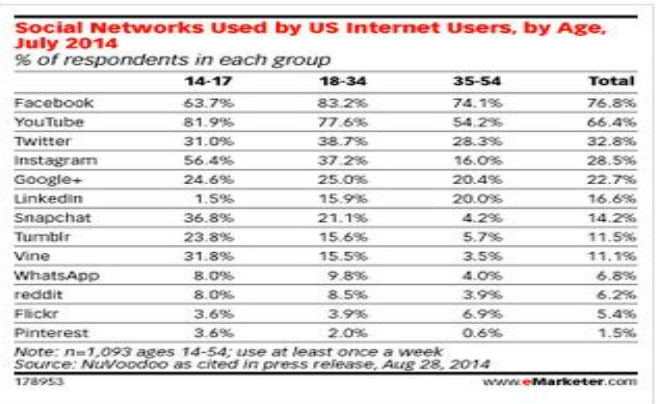

Figure. 1 User pattern of each social media

\section{B. Advantages of Social Media}

Social Media plays an important role in our life. Several merits of social media use include:

- compelling and relevant content finds the attention of future customers and increase brand visibility

- $\quad$ response facility to almost instantly to industry developments and become famous in your field

- $\quad$ it is very cheaper than traditional promotional and advertising activities

- $\quad$ social content can indirectly encourage links to website content by appearing in general search results, improving search traffic and online sales

- $\quad$ deliverance improved customer service and respond effectively to feedback

- $\quad$ customers can find the seller easily through new channels, generating more leads

- $\quad$ improved loyalty and advocacy from the contacted customers [22].

C. Need for Analysing Social Media Data

The use of social media is increasing day by day and this is represented by the no of monthly use as shown in Fig.2. Increasing growth of social media users over internet has also increased their participation in various discussions and activities simultaneously. In case of a product, reviews of users will help to take many important decisions about the services of the product [23]. But manually reading such a bulk amount reviews is a very difficult task. So there is a need of a automatic system which will lead to automatically extract the positive and negative features of the product and make the decision making process easier. There are many sites and companies which perform these activities. 


\section{SENTIMENT ANALYSIS}

Sentiment analysis is a text classification problem, which deals with extracting information present within the text. This extracted information can be then further classified according to its polarity as positive, negative or neutral. It can be defined as a computational task of extracting sentiments from the opinion. Some opinions represent sentiments and some opinions do not represent any sentiment [24].

- Sentiments: Opinions or in other sense can be recognized as someone's linguistic expressions of emotions, beliefs, evaluations etc.

- Analysis: To capture the opinions from a pool of users whether the opinion is positive, negative or neutral.

- $\quad$ Benefit: Provide efficient information in decision making

Example:

User's Opinion: Person a: it's a great movie (positive statement)

Person b: the new iphone is awesome...!!! (Positive statement)

Person c: Nah!! I didn't like it at all.. (Negative statement).

Reading huge amount of reviews and discussions over internet is not an easy task and finally to take decision. But, these discussions and reviews help in many sectors such as improving e-learning environment, providing personalization in e-learning environment, for getting public response to governmental activities [27].

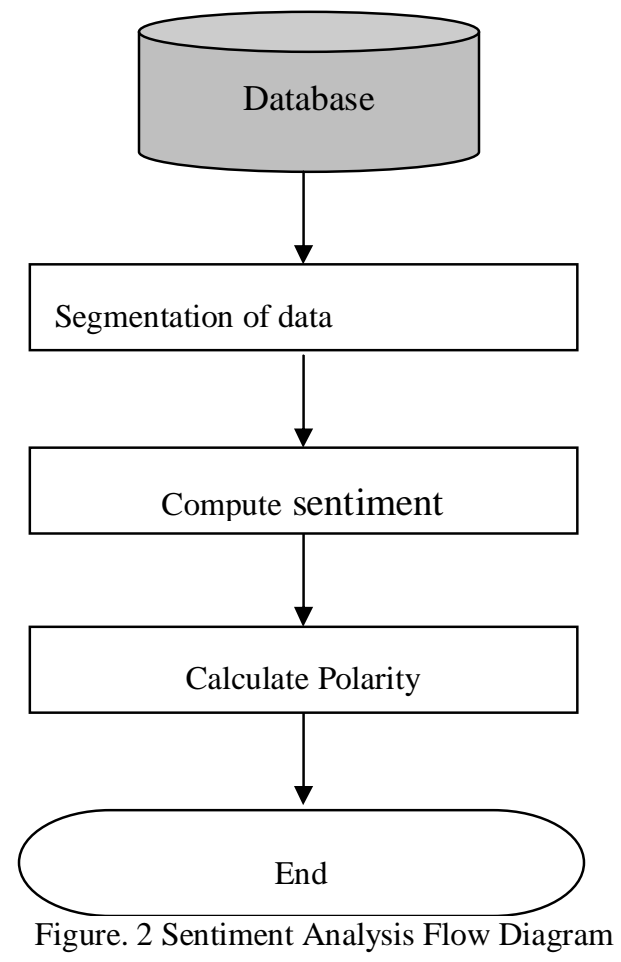

In the Figure .3, flowchart of sentiment analysis is represented which gives the general flow of process of sentiment analysis. From the given dataset, what we have to do is to extract the data and segment that data according to parts of speech. After that we will check the sentiments and assign tags to the extracted tokens. In the last step overall polarity of the text is calculated. If the polarity of data is positive, it is positive sentence and if polarity is negative it is negative sentence. 
A. Applications of Sentiment Analysis

Sentiment examination can be utilized as a part of various fields for different purposes. .

- $\quad$ Online Commerce

- $\quad$ Voice of the Market (VOM) .

- $\quad$ Voice of the Customer (VOC)

- $\quad$ Brand Reputation Management

- $\quad$ Government

\section{FUZZY LOGIC}

Fuzzy logic is the difficult mathematical model for understanding and it gives the uncertainty in reasoning [33]. In the fuzzy logic, the knowledge of experts will be used using IF-THEN rules. A fuzzy logic is a sub- set and whose membership functions are subsets of it. Fuzzy logic mainly depends on the three features, fuzzy values, linguistic variables and probability distribution [33]. Fuzzy logic is conceptually easy to understand. The mathematical concepts behind fuzzy reasoning are very simple. Fuzzy logic is tolerant of imprecise data. Everything is imprecise if you look closely enough, but more than that, most things are imprecise even on careful inspection. Fuzzy reasoning builds this understanding into the process rather than tacking it onto the end [34]. Fuzzy logic is based on natural language. The basis for fuzzy logic is the basis for human communication.

The main feature of fuzzy logic are as follows:

- It contains matter of degree.

- $\quad$ Fuzzy logic is flexible

- $\quad$ Any system can be fuzzified .[35]

- Information is decomposed into collection of variables.

There are five attributes associated with fuzzy expert systems:

1) Input variables,

2) Output variables,

3) Subsets of the inputs and the outputs and the membership functions corresponding to the various subsets leading to fuzzy set [36].

4) Rules connecting the input fuzzy subsets and the output fuzzy subset .[37]

5) Procedure (or methodology) for de-fuzzification of the Output .[38,39,40,41] 


\section{RESEARCH METHODOLOGY}

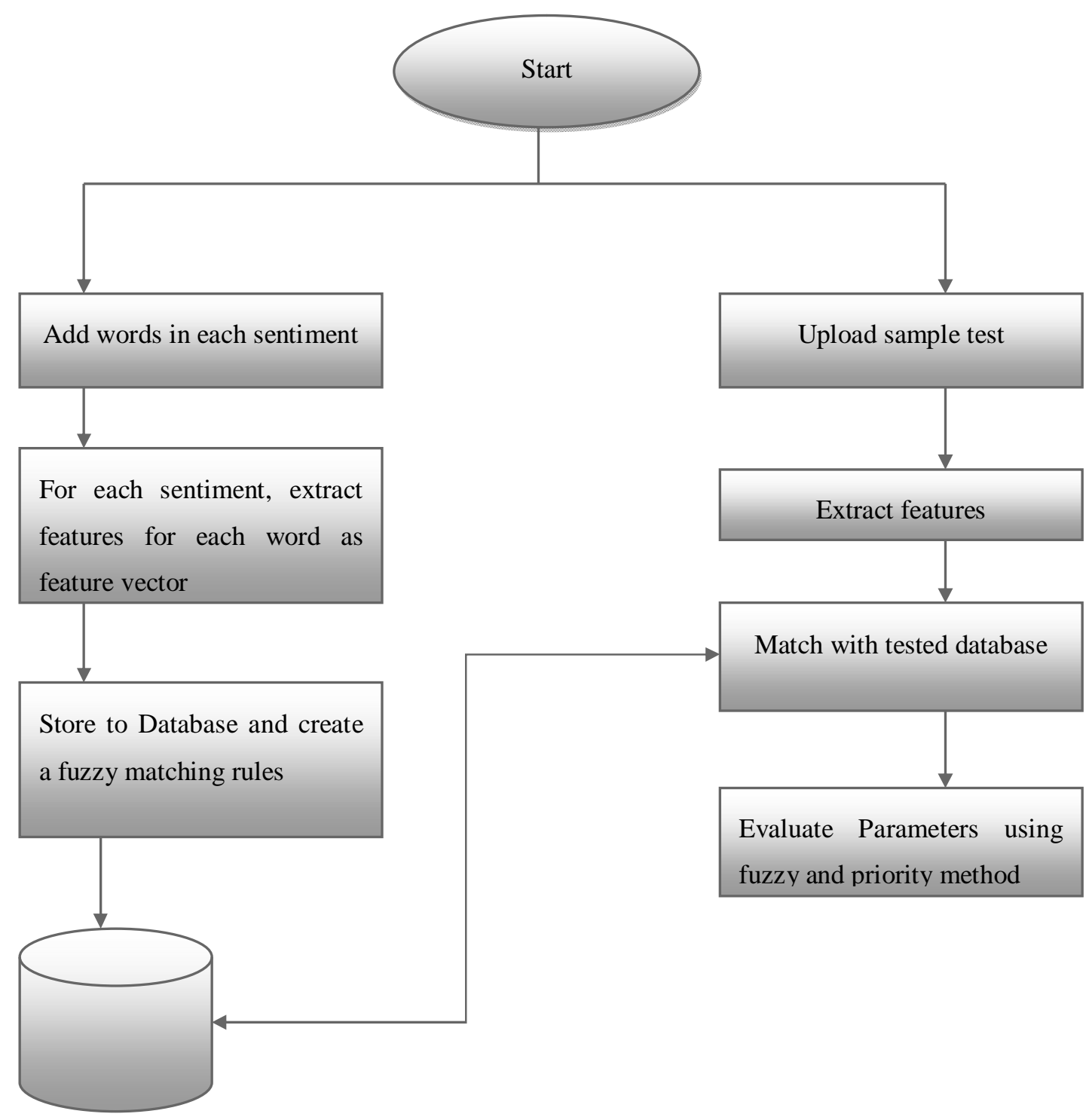

Figure. 4 Steps of Proposed Work

\section{PERFORMANCE EVALUATION}

Performance can be evaluated by these three metrices.

Precision rate : It measure the exactness .Higher precision means less false positives whereas lower precision means more false positives. 
Recall rate: It measures the completeness. Higher recall means less false negative whereas lower recall means more false negatives.

Accuracy: It is defined as the closeness of measured value to actual value

\begin{tabular}{|l|l|l|}
\hline Metrics & Using fuzzy & Using priority \\
\hline Precision rate & .64 & .6 \\
\hline Recall rate & .5 & .75 \\
\hline Accuracy & 54 & 53 \\
\hline
\end{tabular}

Table 1 Result Evaluation

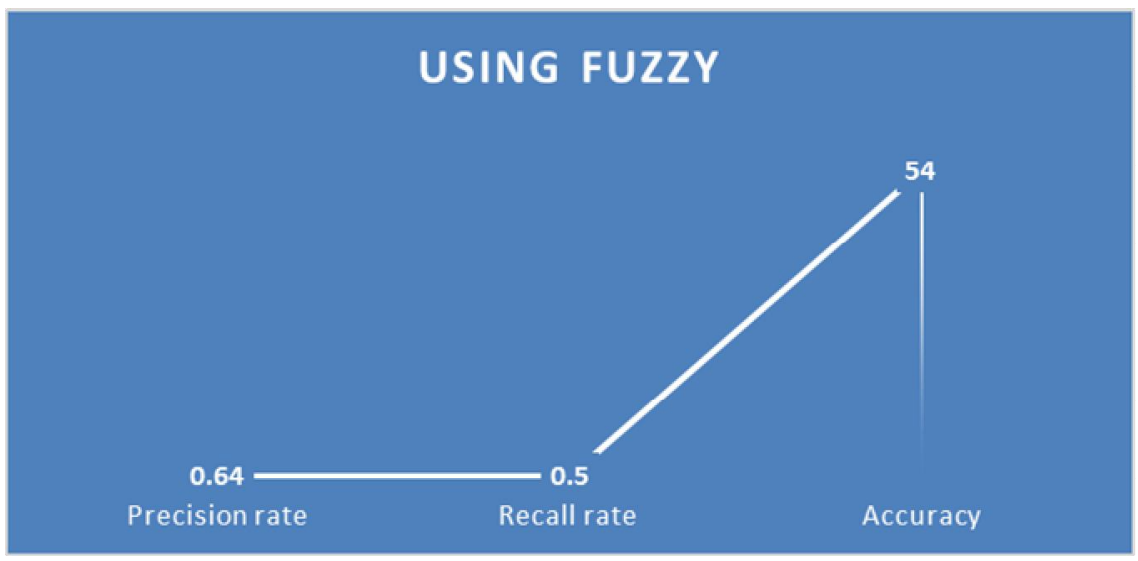

Figure. Error! No text of specified style in document. Result Evaluation using Fuzzy Logic

Graphical representation for fuzzy logic has been presented and the obtained values are accuracy $=54 \%$, recall rate $=.5$, precision rate $=.64$.

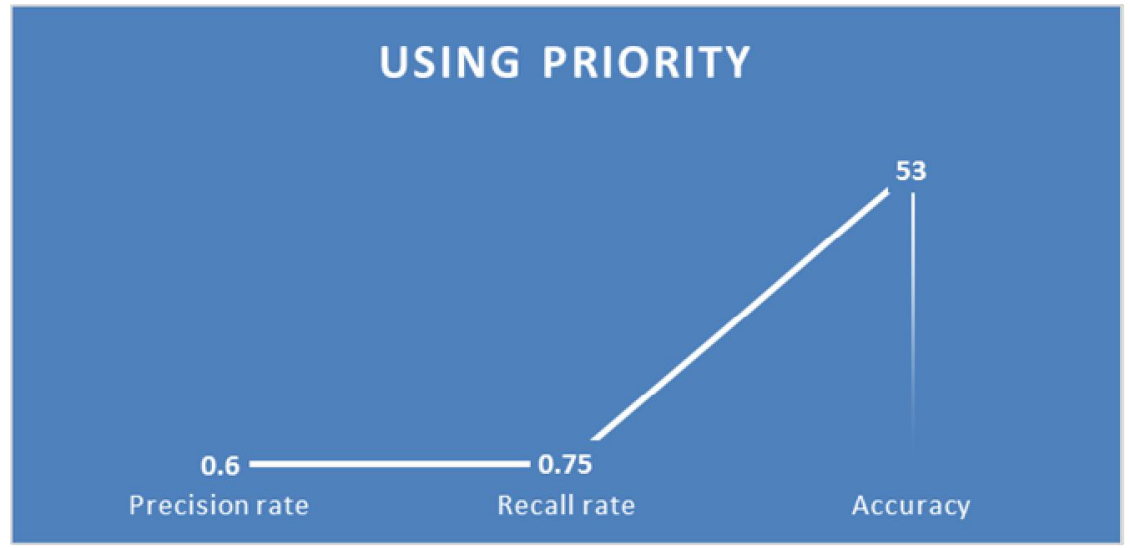

Figure. 6 Result Evaluation using Priority Method

Graphical representation for priority method has been presented and the obtained values are accuracy $=53 \%$, recall rate $=.75$, precision rate $=.6$. 


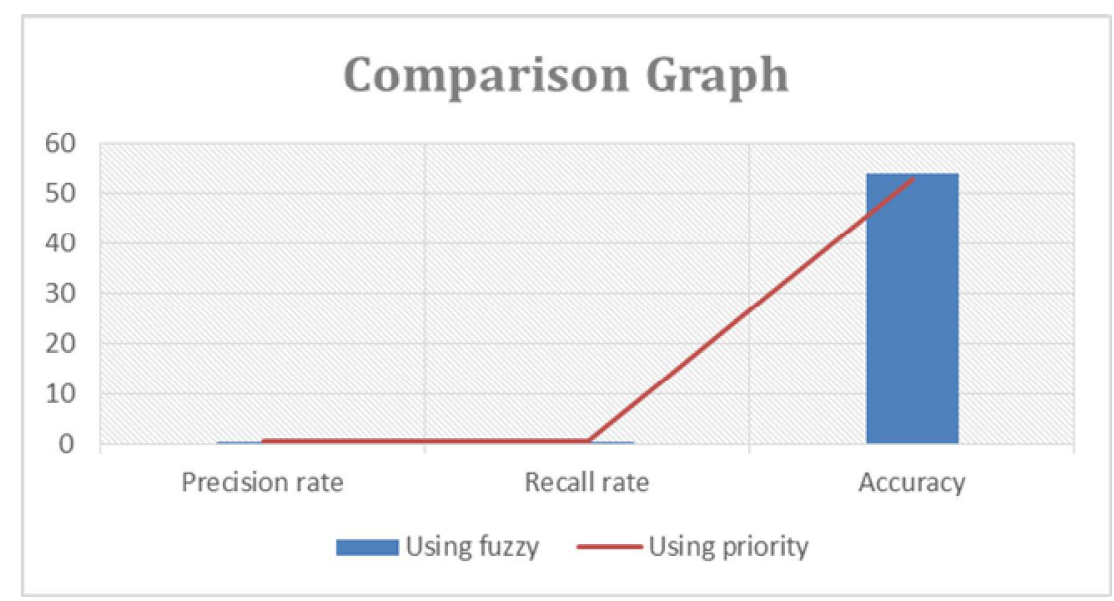

Figure. 7 Comparative Graph Analysis

Comparison graph between fuzzy and priority method has been presented and from result analysis fuzzy logic outperforms having accuracy $54 \%$.

\section{CONCLUSION}

In Social Psychology wherein author clarified the feeling framework and formally arranged the human feelings through a feeling chain of command in six classes at essential level which are Love, Joy, Anger, Sadness, Fear and Surprise. Certain different words additionally fall in optional and tertiary levels. As of late, numerous scientists have concentrated on this region. They are attempting to get sentiment data to examine and abridge the operation opinions communicated naturally with PCs. This new research area is generally called Opinion mining of enormous information and Sentiment Analysis.

Classification problem is one of the problem wherein data has to be classified into different classes present inside the $\mathrm{db}$. This extricated data can be then further arranged by extremity as positive, negative or impartial. It can be characterized as a computational errand of separating notions from the supposition. A few suppositions speak to conclusions and a few assessments do not speak to any opinion. Sentiment analysis is also known as sentiment mining of huge information. SA is the computational investigation of Opinions, conclusions, subjectivity toward an element. The element represents the people, occasions or points. The essential work in the information mining can be arranged in two consequent ways. Initially is called grouping and the other is called making clusters. Information Mining is a term of seeking and investigates of information. Fuzzy logic is thoughtfully straightforward.

Large amount of work has been done in emotion extraction from text. But good accuracy has not been reached by using various techniques of data mining, AI, classification algorithms. So, in proposed work hybridization of both AI and classification algorithm has been done in which fuzzy logic has been utilized. From result simulations it has been concluded that proposed method worked well and showing accuracy of 54\% vis-a-vis to priority method whose accuracy is just $53 \%$.

\section{REFERENCES}

[1] Ortigosa, Alvaro, José M. Martín, and Rosa M. Carro, "Sentiment analysis in Facebook and its application to e-learning", Computers in Human Behavior, 31, pp. 527-541, 2014.

[2] Pak, Alexander and Patrick Paroubek, "Twitter as a Corpus for Sentiment Analysis and Opinion mining of big data”, LREC. Vol. 10, 2010. 
[3] Apoorv Agarwal, Boyi Xie ,Ilia Vovsha,Owen Rambow ,Rebecca Passonneau, "Sentiment analysis of twitter data", Proceedings of the Workshop on Languages in Social Media. Association for Computational Linguistics, 2011.

[4] Fotis Aisopos, George Papadakis, Konstantinos Tserpes, Theodora Varvarigou, "Content vs. context for sentiment analysis: a comparative analysis over microblogs", Proceedings of the 23rd ACM conference on Hypertext and social media. ACM, 2012.

[5] Balahur, Alexandra, et al, "Sentiment analysis in the news", arXiv preprint arXiv:1309.6202, 2013.

[6] Jebaseeli, A. Nisha, and E. Kirubakaran, "A Survey on Sentiment Analysis of (Product) Reviews", International Journal of Computer Applications 47.11 (2012).

[7] Scholar, P. G., "Big-SoSA: Social Sentiment Analysis and Data Visualization on Big Data."

[8] Horakova, Marketa, "Sentiment Analysis Tool using Machine Learning", Global Journal on Technology, 2015.

[9] Gupta, Aditi, et al, "Sentiment analysis for social media", International Journal of Advanced Research in Computer Science and Software Engineering 3.7 (2013): 216-221.

[10] B. Pang and L. Lee, "Opinion mining of big data and sentiment analysis. Foundations and Trends in Information Retrieval”, 2(1 -2):1\{135, 2008.

[11] Mofleh Al-diabat, "Arabic Text Categorization Using Classification Rule Mining", Applied Mathematical Sciences, Vol. 6, 2012, and No. 81, 4033 - 4046.

[12] Dasari, Bhavani, "Text Categorization and Machine Learning Methods: Current State of the Art", Global Journal of Computer Science and Technology, Vol.12 Issue 11 Versions 1.0, 2012.

[13] Pratiksha Y. Pawar and S. H. Gawande, Member, IACSIT, "A Comparative Study on Different Types of Approaches to Text Categorization”, International Journal of Machine Learning and Computing, Vol. 2, No. 4, August 2012.

[14] Megha Dawar1 and Dr.Aruna Tiwari, "Fast fuzzy feature clustering for Text classification", in CS \& ITCSCP, pp. 167-172, 2012

[15] Bhumika, Prof Sukhjit Singh Sehra, Prof Anand Nayyar, "A Review Paper On Algorithms Used For Text Classification", International Journal of Application or Innovation in Engineering \& Management (IJAIEM), Volume 2, Issue 3, March 2013.

[16] Xiaohui Yu, Yang Liu, Jimmy Xiangji Huang, and Aijun, "Mining Online Reviews for Predicting Sales Performance: A Case Study in the Movie Domain", Proc. IEEE Transactions on Knowledge and Data Engineering, VOL. 24, NO. 4, April 2012.

[17] Ghose and P.G. Ipeirotis, "Designing Novel Review Ranking Systems: Predicting the Usefulness and Impact of Reviews," Proc. Ninth Int'l Conf. Electronic Commerce (ICEC), pp. 303-310, 2007.

[18] Pang, L. Lee, and S. Vaithyanathan, "Thumbs Up? Sentiment Classification Using Machine Learning Techniques," Proc. ACL-02 Conf. Empirical Methods in Natural Language Processing (EMNLP), 2002.

[19] Pang and L. Lee, "A Sentimental Education: Sentiment Analysis Using Subjectivity Summarization Based on Minimum Cuts," Proc. 42nd Ann. Meeting on Assoc. for Computational Linguistics (ACL), pp. 271-278, 2004.

[20] Whitelaw, N. Garg, and S. Argamon, "Using Appraisal Groups for Sentiment Analysis," Proc. 14th ACM Int'l Conf. Information and Knowledge Management (CIKM), pp. 625-631, 2005.

[21] N. Archak, A. Ghose, and P.G. Ipeirotis, "Show Me the Money: Deriving the Pricing Power of Product Features by Mining Consumer reviews,'Proc.13thACM SIGKDD Int'l Conf Knowledge Discovery and Data Mining (KDD), pp. 56-65, 2007.

[22] N. Jindal and B. Liu, "Opinion Spam and Analysis," Proc. Int'1 Conf. Web Search and Web Data Mining (WSDM), pp. 219-230, 2008.

[23] J. Liu, Y. Cao, C.-Y. Lin, Y. Huang, and M. Zhou, "Low Quality Product Review Detection in Opinion Summarization," Proc. Empirical Methods in Natural Language Processing and Computational Natural Language Learning (EMNLP), pp. 334-342, 2007

[24] Ortigosa, Alvaro, José M. Martín, and Rosa M. Carro, "Sentiment analysis in Facebook and its application to e-learning", Computers in Human Behavior 31 (2014): 527-541.

[25] Pak, Alexander, and Patrick Paroubek, "Twitter as a Corpus for Sentiment Analysis and Opinion mining of big data”, LREC. Vol. 10. 2010.

[26] Agarwal, Apoorv, et al, "Sentiment analysis of twitter data." Proceedings of the Workshop on Languages in Social Media", Association for Computational Linguistics, 2011.

[27] Aisopos, Fotis, et al, "Content vs. context for sentiment analysis: a comparative analysis over microblogs", Proceedings of the 23rd ACM conference on Hypertext and social media. ACM, 2012.

[28] Balahur, Alexandra, et al, "Sentiment analysis in the news", arXiv preprint arXiv: 1309.6202, 2013.

[29] Jebaseeli, A. Nisha, and E. Kirubakaran, "A Survey on Sentiment Analysis of (Product) Reviews", International Journal of Computer Applications 47.11 (2012).

[30] Scholar, P. G, "Big-SoSA: Social Sentiment Analysis and Data Visualization on Big Data." 
[31] Horakova, Marketa, "Sentiment Analysis Tool using Machine Learning”, Global Journal on Technology, 2015.

[32] Asst. Prof. A Kowcika , Aditi Gupta, Karthik Sondhi, Nishit Shivhre, Raunaq Kumar, "Sentiment analysis for social media", International Journal of Advanced Research in Computer Science and Software Engineering 3.7 (2013): 216-221.

[33] Nguyen, H. T. and Walker, E., "A First Course in Fuzzy Logic”, CRC Press, Boca Raton, 1997.

[34] Nguyen, H. T., Sugeno, M., Tong, R. M., and Yager, R. R., "Theoretical Aspects of Fuzzy Control”, 1995.

[35] John Wiley \& Sons, New York. Novak, V, "Fuzzy Sets and Their Applications", 1989.

[36] Adam-Hilger, Bristol. Olaru, C. and Wehenkel, L, "A complete fuzzy decision tree technique", Fuzzy Sets and Systems, 138(2):221-254, 2003.

[37] Pedrycz, W., "Fuzzy Control and Fuzzy Systems", 1989.

[38] Vieweg, Braunschweig. Tizhoosh, H. R., "Fuzzy-Bildverarbeitung", Einf "uhrung in Theorie und Praxis. Springer, Berlin, 1998.

[39] Zadeh, L. A, "Fuzzy sets", Inf. Control, 8:338-353, 1965.

[40] Zadeh, L. A., "Outline of a new approach to the analysis of complex systems and decision processes", IEEE Trans. Syst. Man Cybern., 3(1):28-44. Zimmermann, 1973.

[41] H.-J., "Fuzzy Set Theory-and its Applications", Kluwer Academic Publishers, Boston, second edition, 1991. 